\title{
Experiencia y
}

esperanzas docentes

en la educación

virtual

Xavier Ernesto Rodriguez Corea 


\section{Experiencia y esperanzas docentes en la educación virtual \\ Experiences and educational \\ expectations in virtual education \\ Xavier Ernesto Rodríguez Corea Docente investigador \\ Departamento de Antropología Fac. de Humanidades y Ciencias Jurídicas UNAN-Managua ORCID. 000-0002-2335-2477 xrodriguez@unan.edu.ni}

\section{RESUMEN}

La educación virtual es un ámbito educativo que como país y como institución educativa estamos desarrollando. Sin embargo, por ser un proceso reciente y con muchas incertidumbres, es necesario compartir estas reflexiones. En este sentido partimos de reflexionar sobre nuestro papel como docentes en esta experiencia y las esperanzas de que esta sea la oportunidad para fortalecer los deseos de otra educación posible, mas encarnada en el mundo actual y en el lenguaje nativo de las nuevas generaciones.

\section{ABSTRACT}

Virtual education is an educational field that we are developing as a country and educational institution. However, as it is a recent process with many uncertainties, it is necessary to share these reflections. In this sense, we start by reflecting on our role as lecturers in this experience and the expectations that this is the opportunity to reinforce the desires for another possible education, more incarnated in this contemporary world, as well as in the native language of the new generations.

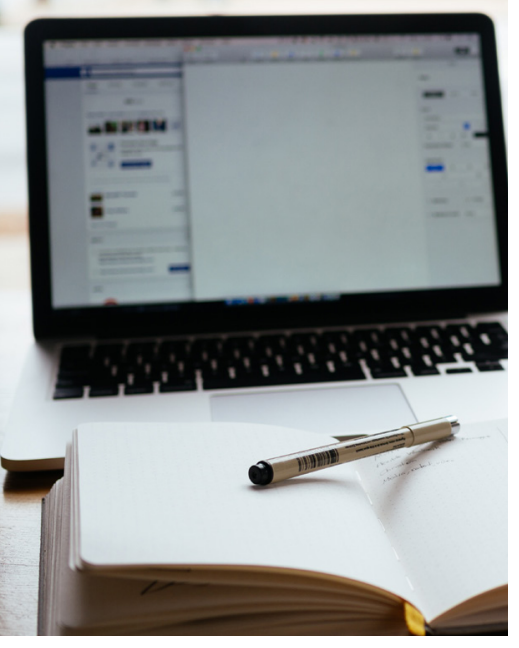

PALABRAS CLAVE

Educación virtual, virtualización, Nicaragua, Universidad

\section{KEY WORDS}

Virtual education, virtualization, Nicaragua, University 


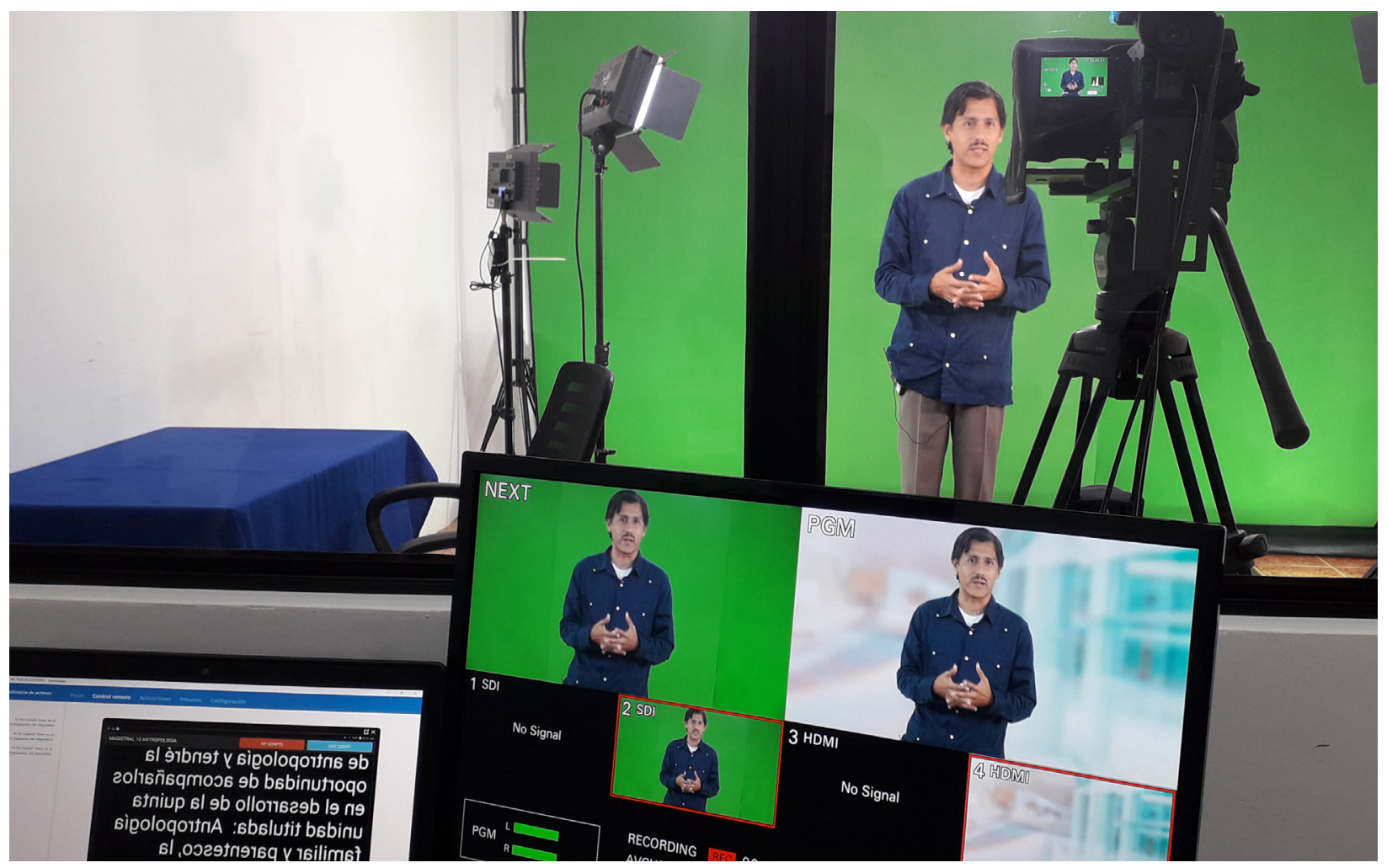

\section{Introducción}

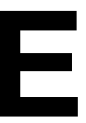

ste ensayo busca reflexionar desde nuestra experiencia; es decir, es una reflexión sobre pasos y procesos concretos que hemos dado en el Departamento de Antropología, en torno a la virtualización de la asignatura Introducción a la Antropología. Esta asignatura actualmente en modalidad regular por encuentro es inscrita por un promedio de 1500 estudiantes, pues es una asignatura optativa en alrededor de 25 grupos de clase en el Recinto Universitario Rubén Darío (RURD) de la Universidad Nacional Autónoma de Nicaragua, UNAN-Managua.

Esta asignatura es el resultado de un trabajo articulado de alrededor de 10 docentes que semestre a semestre adecuamos, desde la experiencia vivida, la metodología de trabajo en cátedras magistrales y subgrupos.

Aclarado esto, es necesario recalcar que los próximos párrafos no deben leerse como una receta, tampoco como las apreciaciones de un especialista en el tema de virtualización. Esta reflexión trata de ser espejo donde otros que están inmersos en procesos de cambio a la educación virtual, que no están, pero piensan estar, o están, pero quisieran no estar; cualquiera sea el caso, espero en base a nuestra experiencia, se vean reflejados en nuestras reflexiones y ayude a mejorar estos procesos, complejos, urgentes y necesarios.

Otra aclaración es que, aunque fue escrito por una sola persona, las reflexiones aquí vertidas son producto de un proceso de interrelación, conversación - diálogo y trabajo con otras muchas personas. La conversación ha parido, consolidado y reafirmado ideas que aquí comparto. La conversación y el diálogo son la base de la entrevista en términos de la Antropología social, pero sobre todo de aquella práctica propuesta por los zapatistas de caminar preguntando, tan útil en los procesos educativos para combatir la inflamación del ego académico y fermento de la verdadera interdisciplinariedad. 


\section{Nicaragua en la era de las nuevas tecnologías}

Durante los últimos años, Nicaragua ha dado pasos de gigantes en lo que respecta del acceso a las nuevas tecnologías y concretamente en el acceso al internet. Según datos de CEPAL, en el informe sobre Estado de la banda ancha en América Latina y el Caribe 2016, "los países que tuvieron las mayores tasas de crecimiento del número de hogares conectados a Internet en 2010-2015 fueron Nicaragua, Guatemala, El Salvador y el Estado Plurinacional de Bolivia, países que tenían muy baja tasa de penetración al inicio del periodo" (CEPAL NU, 2016). Este informe refleja el crecimiento exponencial de Nicaragua en el acceso al internet en los últimos años, datos que coinciden con los vertidos por la Cámara Nicaragüense de Internet y Telecomunicaciones (CANITEL) en conferencia de prensa y recogidos por el portal El 19 Digital, según los cuales, "el $100 \%$ de las cabeceras departamentales tengan acceso a la telefonía convencional, móvil e internet. Igual existen otras 3 mil 500 comunidades rurales que están conectadas al igual que unos 2 mil 500 kilómetros de las principales carreteras del país" (Ramírez, 2016).

Estos datos pudieran ser solo eso, si no logramos mirar a nuestro alrededor y comprobar que efectivamente la vida cotidiana de las poblaciones urbanas y rurales de buena parte de Nicaragua ha cambiado significativamente en los últimos años. La conexión a internet y el acceso a nuevas tecnologías, entre ellas el celular resultan evidentes, no podemos afirmar aún que este acceso se traduce en un mayor uso para el ámbito educativo, pues por lo general y contrario a otros medios tradicionales como la radio o los medios escritos, el internet nació con un fuerte sesgo recreativo más que informativo.

En el caso de Nicaragua, este impulso en el acceso al internet está mediado por el papel del Estado. En este ámbito, las políticas gubernamentales son un referente a nivel centroamericano. Una de las medidas gubernamentales es la mejora de los espacios públicos y en especial los parques. Esto es, sin lugar a dudas, un cambio sustancial, pues durante los gobiernos neoliberales de los años 90 y principios del 2000, se había dejado la recreación como un producto solo al alcance de la clase media y la clase alta. Junto a esta mejora en la infraestructura de los parques de las comunidades rurales y los sectores populares de las ciudades llegó también el internet libre en estos espacios públicos, convirtiendo el acceso a internet en un derecho de buena parte de la población que antes tenía este servicio censurado de forma fáctica por la dictadura del mercado y la inoperancia de los gobiernos que hacía poco o nada por masificar el servicio.

La política gubernamental de acceso a las telecomunicaciones y al internet no podemos desvincularla de otras como la creación de la Universidad Abierta en Línea de Nicaragua (UALN), un proyecto liderado por la propia presidencia de la República y ejecutado por el Consejo Nacional de Universidades (CNU). En el año 2018 la UALN ofertó 13 programas de cursos libres, 15 técnicos superiores, 14 licenciaturas y 4 posgrados.

En este sentido, Nicaragua se posiciona como uno de los países donde la educación virtual es hoy una realidad palpable y en desarrollo, no un proyecto a futuro como refiere Manuel Castells, director del Internet Interdisciplinary Institute en la Universidad Abierta de Cataluña. El internet es algo más que una herramienta como otras del desarrollo de la sociedad:

"Internet es el tejido de nuestras vidas en este momento. No es futuro. Es presente. Internet es un medio para todo, que interactúa con el conjunto de la sociedad y, de hecho, a pesar de ser tan reciente, en su forma societal (aunque como sabemos, Internet se construye, más o menos, en los últimos treinta y un años, a partir de 1969; aunque realmente, tal y como la gente lo entiende ahora, se constituye en 1994, a partir de la existencia de un browser, del World Wide Web) no hace falta explicarlo, porque ya sabemos qué es Internet. Simplemente les recuerdo, para la coherencia de la exposición, que se trata de una red de redes de ordenadores capaces de comunicarse 
entre ellos. No es otra cosa. Sin embargo, esa tecnología es mucho más que una tecnología. Es un medio de comunicación, de interacción y de organización social." (Castells, 2001, p. 1)

\section{Educación virtual: Una reflexión desde nuestra experiencia}

La aventura de la educación virtual nos llegó al Departamento de Antropología en medio de una gran cantidad de otras tareas prioritarias importantes; sin embargo, ya en nuestra cabeza rondaba la idea de la educación virtual desde aspectos incipientes como la creación de un dossier digital de los materiales de la asignatura que incluyera además de textos, audios, videos y en algunos casos el uso de la plataforma Moodle en curso de la licenciatura en Antropología Social. En el caso del posgrado, el desarrollo de curso a distancia con el apoyo de las video conferencias. Todas estas experiencias muchas veces se desarrollaron con mayor o menor éxito. Sin embargo, emprendimos la aventura, pensado que, aunque son innumerables las cosas urgentes, solo algunas resultan tan urgentes e importantes en estos momentos del desarrollo del país.

Estamos viviendo un cambio de época y con esto de paradigmas en lo que respecta a la educación-comunicación. No me refiero a las teorías y propuestas de la pedagogía, sino más bien a las concepciones desde la vida cotidiana que podemos estudiar desde la Antropología con respecto a la educación en términos amplios, no solo reducidos a los espacios educativos formales como la escuela y la universidad, sino abiertos a los procesos educativos y de comunicación que hoy están vinculados profundamente a los medios y en especial a internet. Se trata de cambios que trastocan todos los ámbitos de la vida y a los cuales debemos hacerle frente, para que no sean dirigidos por lo invisible del mercado, sino más bien por nosotros mismos y nuestras intenciones como país.

El programa de la UNAN-Managua, Universidad en el Campo (UNICAM), verifica la necesidad de que la universidad se mueva hacia la población que necesitan tecnificarse -profesionalizarse. Tomando como antecedentes este programa, la educación virtual podría maximizar el alcance de la educación superior en Nicaragua. Estoy seguro que en los próximos años veremos un incremento en las cifras relacionadas con los estudios superiores en Nicaragua y este incremento directamente reflejado en la educación virtual.

El traslado de la educación al ámbito virtual implica llevar la educación a los ámbitos nativos de las nuevas generaciones; no obstante, para muchos docentes es salir de nuestra "zona de confort" y trasladar nuestra tienda a un mundo poco conocido. Esto implica en los docentes una actitud de apertura al cambio y adaptación a las nuevas realidades, retomando algunos principios e ideas y descartando otras que en la educación virtual nos damos cuenta, no funcionan.

\section{Virtualizar e internacionalizar los entornos educativos}

El acceso a la información por medio del internet ha abierto un mundo de posibilidades y de retos que deben ser asumidos no como una amenaza, sino como una oportunidad. El espacio virtual facilita una serie de posibilidades que son completamente coherentes con nuestras realidades latinoamericanas y concretamente como universidad. Por ejemplo, el 2018 se nombró en la UNAN-Managua el Año de la Internacionalización de la Universidad. El internet ha sido hasta la fecha un recurso importante para lograr avances significativos en el área de internacionalización. Un pasaje aéreo para cualquier país de Latinoamérica representaría recursos monetarios que son urgentes para otros menesteres, especialmente en una universidad pública que responde a las amplias necesidades de nuestro pueblo. Desde la experiencia de la Maestría en Antropología y Liderazgo Social, III Cohorte, hemos desarrollado el curso de Liderazgo II a cargo del profesor colombiano Luis Felipe Ulloa, en las dos primeras ocasiones desde Brasil y en esta última 
ocasión desde Colombia. El curso resultó un éxito, la plataforma Webex, una buena conexión, el apoyo técnico de los especialistas y seguimiento en presencial desde la coordinación de la maestría aseguran un espacio educativo muy dinámico y de alto nivel.

Esta posibilidad de optimización de los recursos y de ampliación de la cobertura en los espacios educativos requiere desarrollar nuestra habilidad en el manejo de la plataforma y recursos necesarios. Nuestra universidad invierte en equipos y aplicaciones que aún seguimos utilizando de forma tradicional. Es necesario tomar iniciativas desde los distintos niveles para potenciar el uso efectivo de las plataformas de comunicación, equipos técnicos, base de datos y todas las plataformas necesarias.

\section{Re-educarnos hacia lo virtual}

La educación virtual implica un primer momento de re-educación de quienes trabajamos en el ámbito educativo. El diseño de espacios de educación virtual implica algo más que un mero conocimiento técnico de las herramientas virtuales. Es ante todo un cambio de lógica de la educativa presencial, es también una oportunidad de sacudirnos los muchos o pocos grilletes de la educación bancaria (Freire, 2012).

La educación virtual posibilita actividades que generen menos dependencia del docente en términos presenciales y con esto la auto-gestión y la administración del tiempo por parte de los participantes.

Educación virtual es ante todo una oportunidad para los educadores de re-aprender a enseñar, para re-pensar nuestras actividades, nuestro rol e incluso nuestros contenidos; en otras palabras, re-pensar el aprendizaje a como lo refiere José Antonio Blanco: "El aprendizaje es una acción biológica fundamental, y se saca esto a colación porque en la "Institucionalidad Educativa" se ha circunscrito a construcciones cognitivas derivadas de una enseñanza lineal y adoctrinadora (Blanco, 2014, pág. 5)

Los docentes tenemos la tarea urgente de sacar el pie que hemos metido poniéndonos al centro del proceso. Esto genera dependencia a la espera de la presencia paternal-maternalcontroladora- castigadora del educador con un tiempo y un espacio determinados. Virtualizar nuestras clases es cambiar la lógica de trabajo docente, promover la educación para la libertad que genera el placer que debe reinar en nuestros procesos educativos. Nunca podremos desarrollar la educación virtual bajo la lógica del valle de lágrimas con que muchas veces los docentes asumimos los espacios educativos. Aunque no lo expresamos, aún sigue en nuestra mente la idea de que "las letras con sangre entran". Como he apuntado anteriormente, estamos mudando nuestras tiendas al territorio nativo de muchos de los participantes, las nuevas generaciones han usado el internet para aprender -divirtiéndose.

Otra lección aprendida está relacionada con la importancia de los audiovisuales en los nuevos procesos educativos como herramienta autodidáctica. Las redes sociales que son las nuevas formas de socialización han logrado que las nuevas generaciones y buena parte de las generaciones anteriores se sumen de forma muy entusiasta a largos periodos de aprendizaje informal, principalmente mediado por los audiovisuales. La red social Facebook, de mayor uso en nuestro país, ha migrado rápidamente del texto a la imagen y al video; Twitter, de menor uso en Nicaragua, ha visto cómo sus publicaciones de caracteres son cada día más invadidos por imagen y videos. 


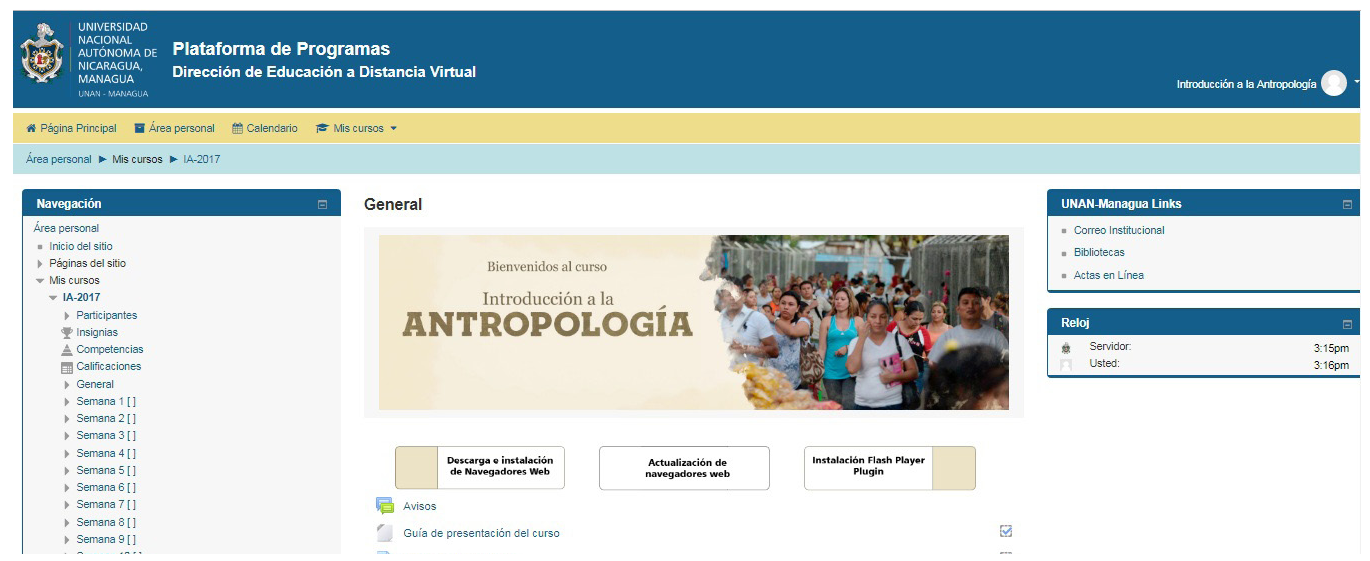

En nuestra experiencia concreta hemos visto que para los grupos de clase un vídeo dice más que mil palabras. Esto ha requerido el desarrollo de la habilidad, no sin tropiezos, de crear, buscar y usar adecuadamente elementos audiovisuales atractivos y funcionales.

La imagen en cualquier espacio educativo es importante, pero en la educación bancaria (Freire, 2012) fue sustituida por la dictadura de la palabra. La educación virtual nos pone el reto, pero además la oportunidad de usar al ámbito visual en nuestras actividades y lógicas que calza perfectamente con la lógica de las nuevas generaciones, donde lo visual juega un papel central en todos los ámbitos de la vida cotidiana. Así, por ejemplo, una infografía puede ser la compañera perfecta de un documento que explique la ruta temática y metodológica de la clase. Ahora bien, no se trata de borrar las palabras y el texto, sino más bien de reubicarlo en un sitio donde comparta centralidad con el aspecto visual - gráfico.

\section{Conclusiones}

Aunque estamos dando nuestros primeros pasos en torno a la educación virtual, las condiciones como país y a nivel institucional son las idóneas para el desarrollo de este nuevo modelo educativo que ampliaría la cobertura del sistema educativo, lo cual a su vez sería trascendental para el desarrollo humano.

El desarrollo de este modelo educativo nos reta a asumir este desafío como una tarea de la sociedad, pero buena parte de la responsabilidad recae en las los docentes de las instituciones educativas públicas. Nuestra posibilidad de adaptarnos y mudar nuestras tiendas es fundamental en la consolidación de la educación virtual.

La adecuada gestión de los recursos, la actitud abierta al cambio y la voluntad política desde el Estado y desde nuestra institución educativa potencian nuestras posibilidades para desarrollar de manera exitosa la educación virtual.

La educación virtual nos posibilita pensar la educación y el aprendizaje, incluyendo en esto, nuestro rol y las lógicas educativas que hasta hoy hemos desarrollado. Este reto no es fácil, salir de la "zona de confort" es una tarea difícil; sin embargo, sabemos que es la que nos ha posibilitado dar los más grandes y hermosos pasos que como sociedad hemos experimentado. 


\section{Bibliografía}

- Blanco, J. A. (2014). Educación virtual no es solo gestión educativa. Senderos Pedagógicos, 5.

- $\quad$ Castells, M. (2001). Internet y la sociedad red. La factoría. La factoría, 15.

- CEPAL NU. (2016). Estado de la banda ancha en América Latina y el Caribe 2016. Santigo, Chile : NU .

- Freire P. (2012). Pedagogía del Oprimido. Siglo XXI Editores: España

- Ramírez, P. O. (11 de agosto de 2016). Destacan crecimiento de las telecomunicaciones y el internet en Nicaragua. El 19 digital.

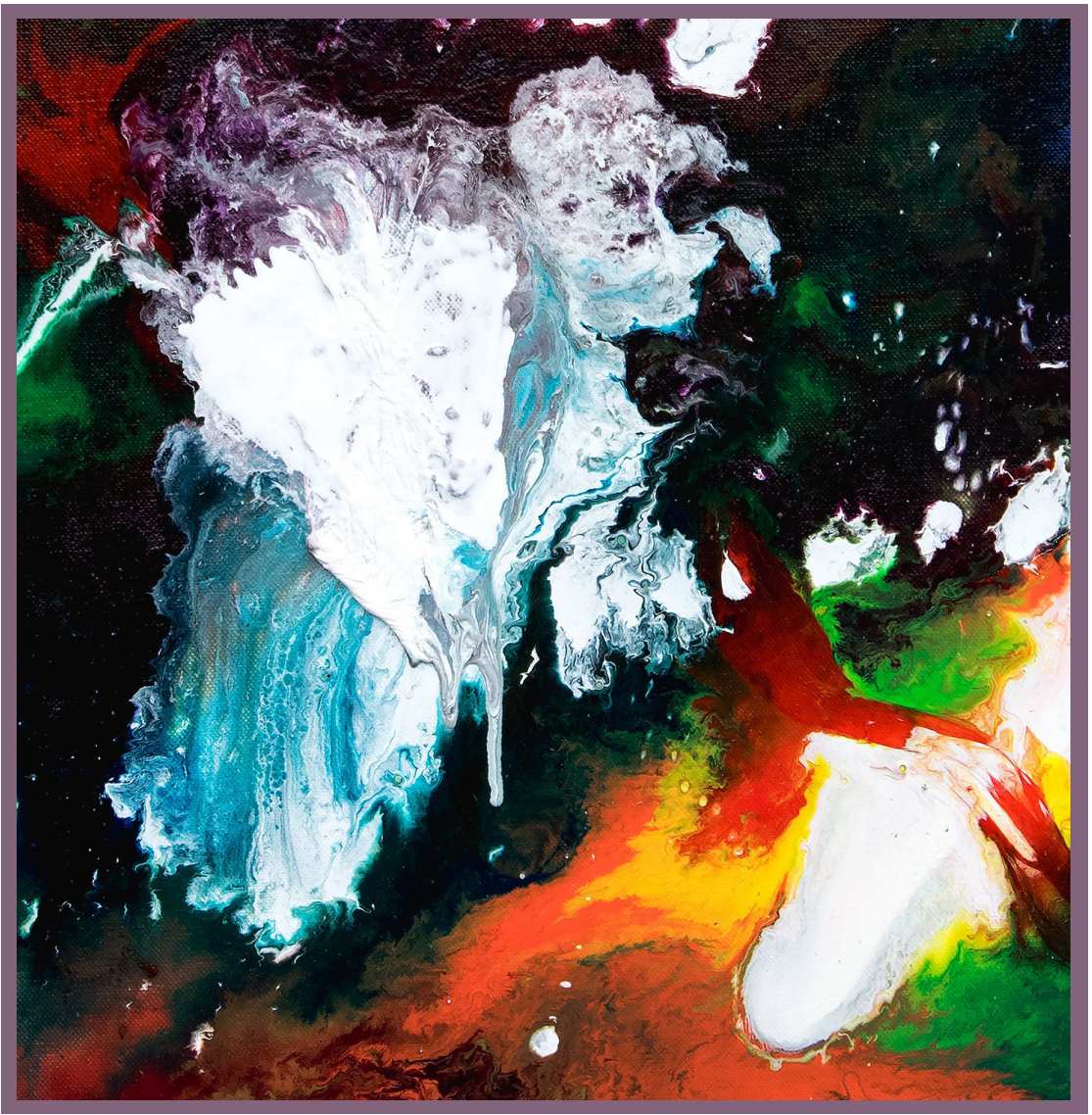

Arte de Lonfeldt 